\title{
Analysis of the motion characteristics of Marine Mecanum transport platform
}

\author{
Zhang Ying ${ }^{1,}$ Ge Shengli, ${ }^{1,}$ \\ ${ }^{1}$ Zheng zhou Electromechanical Engineering Research Institute, Zheng zhou China
}

\begin{abstract}
This paper analyzes the shortage of unmanned systems in the traditional operation mode from the operational requirement of future war to unmanned systems, and puts forward the ship Mecanum transport platform.
\end{abstract}

\section{Introduction}

In the future war, various types of military unmanned equipment will emerge in large numbers as the widespread application of the unmanned platforms. Due to the limited transportation space and narrow workplace channel in the ship deck transportation system, traditional wheeled transport equipment consists of ordinary wheels system and corresponding steering system, which has many disadvantages, such as large turning radius, low utilization rate of space, poor mobility and low transfer efficiency. These adverse factors have seriously affected the work efficiency of the ship's material security system. While the comprehensive motion technology based on the Mecanum wheel can effectively solve this problem. Omnidirectional devices applied this technology can generate 3DOF omnidirectional motion in two-dimensional plane. The omnidirectional transport vehicle loaded by the Mecanum wheel can reach the target location along any path. There is no steering structure of the ordinary power vehicle, and there is no steering wheel, while the desired body movement can be achieved only by the handle operation, especially in the narrow or crowded situations, it still has a much better flexible movement characteristics than other tradition sports equipment. At the same time, this technology has also effectively solved the problem of the transportation of long material in narrow spaces. The schematic diagram is shown in Figure 1.

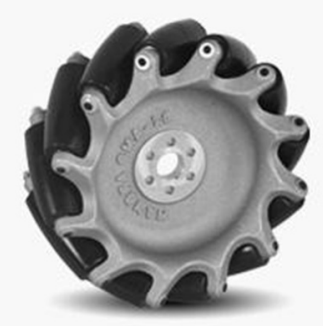

Figure 1 Mecanum wheel

\section{Analysis of the Platform Structure Base on the Mecanum Wheel}

\subsection{The Establishment of the Mathematical Model of Mecanum Wheel}

Several parameters such as radius $\mathrm{R}$, width $\mathrm{b}$, roller length $\mathrm{L}$, number of rollers $\mathrm{N}$, offset angleaand coincidencezare included in designing a complete Mecanum wheel. According to the geometry of the Mecanum wheel, the description of individual wheel's roller coordinates is shown in figure 2.

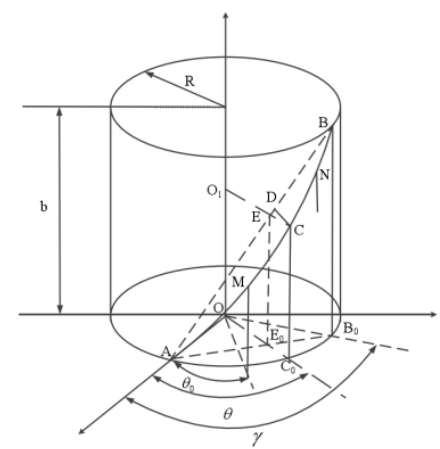

Figure 2 Coordinate Diagram of the Mecanum Wheel Roller

In Figure 2, set the bottom plane's center $\mathrm{O}$ as the origin, wheel axis as the $\mathrm{Z}$-axis, and establish the $\mathrm{O}$-xyz system. Circle $\mathrm{O}$ is the theoretical circle, $\mathrm{R}$ is the radius and line $A B$ is the roller axis, $\alpha$ is angle between $z$-axis and roller axis $\mathrm{AB}$, which is the offset angle. Curve $\mathrm{AB}$ is the contact line between the normal rolling wheel and the ground, and it is also the theoretical bus bar for generating roller bodies. $\mathrm{C}$ is the ground point, yand $\beta$ are the central angled of the projection of the curve $\mathrm{AB}$ and $\mathrm{AC}$ in surface $\mathrm{O}$-xy. The width of wheel is $\mathrm{b}=2 \mathrm{R} \sin \left(\frac{\gamma}{2}\right)$, point $\mathrm{E}$ at the line $\mathrm{AB}, \mathrm{CE} \perp \mathrm{AB}$, the length of $\mathrm{CE}$ is $\delta$, from figure 2 we know that : 


$$
\delta=\mathrm{R} \sqrt{\begin{array}{l}
\left(-\frac{\sin \theta}{\cos \frac{2 \theta-\gamma}{2}}\right)^{2}+4 \sin \frac{\theta}{2}- \\
2\left(\sin \frac{\theta}{2} \cos \frac{\theta-\gamma}{2}+\frac{\sin \theta}{\cos \frac{2 \theta-\gamma}{2}}\right)^{2}
\end{array}}
$$

In $\mathrm{O}$-xyz plane, the point-wise equation of sub-axis $\mathrm{AB}$ is

$$
\frac{x-R}{R(\cos \gamma-1)}=\frac{y}{R \sin \gamma}=\frac{z}{2 R \sin \left(\frac{\gamma}{2}\right)}
$$

According to the Ilon design principle, the envelope of the roller is a complete circle which satisfy the following:

$$
\mathrm{N}\left(\gamma-2 \theta_{0}\right) \geq 2 \pi
$$

If and only if the equal sign is used in Equation 2.1, the linear roller length reaches its minimum:

$$
L_{\text {min }}=2 R \frac{\sin \left(\frac{\gamma-2 \theta_{0}}{2}\right)}{\sin \alpha}=2 R \frac{\sin \left(\frac{\pi}{N}\right)}{\sin \alpha}
$$

We can refer to a pair of involute cylindrical gear continuous transmission conditions that $\varepsilon_{\alpha} \geq\left[\varepsilon_{\alpha}\right]$ $\mathcal{E}_{\alpha}$ is the gear coupling coincidence degree, $\left[\mathcal{E}_{\alpha}\right]$ is the allowable degree of coincidence of gear transmission, generally set $\left[\varepsilon_{\alpha}\right]$ to 1.4 ). In order to ensure smooth transmission, the length $\mathrm{L}$ of the roller in Mecanum wheel is in range of:

$$
L_{\min } \leq L \leq \sqrt{2 R^{2}-2(R-\delta)^{2}}
$$

Therefore, the coincidence degree of Mecanum can be presented as

$$
\boldsymbol{\varepsilon}=\frac{L}{L_{\min }}
$$

In conclusion, the structure parameters of Mecanum wheel are listed in Table 2.1

Table2.1 The Mecanum wheel's structure parameters

\begin{tabular}{cccccc}
\multicolumn{6}{c}{ structure parameters } \\
\hline $\mathrm{R}$ & $\mathrm{b}$ & $\mathrm{L}$ & $\mathrm{N}$ & $\alpha$ & $\varepsilon$ \\
64.1 & 45.7 & 39.2 & 15 & 45 & 1.4
\end{tabular}

Separate components of the wheel designed from the above parameters are shown in Figure3:
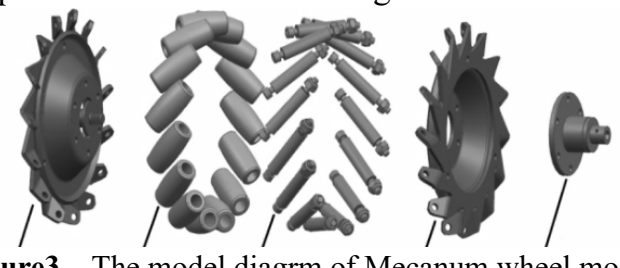

Figure3 The model diagrm of Mecanum wheel model

\subsection{The Establishment of the Unmanned platform 3D structure}

The unmanned platform motion actuator based on the Mecanum wheel is mainly composed of four Mecanum wheels, reducer, platform chassis and four DC motors. The four Mecanum wheels of the platform are respectively controlled by four motors and are mutually independent in the working process to achieve the various movement states of the platform. The simplified model is shown in Figure 4:

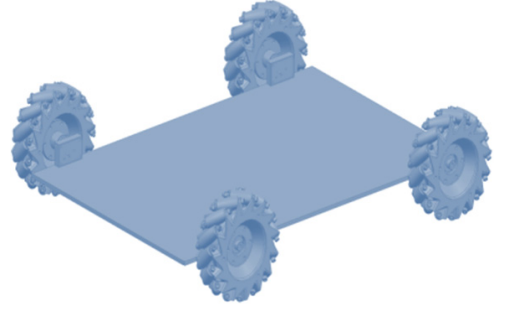

Figure 4 The simplified model of Unmanned platform

According to the characteristics of geometrical parameters of Mecanum wheel, from the consideration of torque and speed, a decelerator with a reduction ratio of 20 is designed. Based on the overall size requirements, design the unmanned platform chassis.

Table2.2 Main parameter table for unmanned platform of rectangular chassis

\begin{tabular}{cccc}
\hline length/mm & 700 & radius/mm & 129 \\
\hline width/mm & 500 & $\begin{array}{c}\text { Valid } \\
\text { width/mm } \\
\text { high/mm }\end{array}$ & 48 \\
Wheel base/mm & 116 & $\begin{array}{c}\text { Motor model } \\
\text { power(DC)/V }\end{array}$ & 245 \\
tread/mm & 425 & speed/rpm & 1000 \\
weight/Kg & 470 & $\begin{array}{c}\text { torque } / \mathrm{Kg} . \mathrm{cm} \\
\text { Rotor } \\
\text { current/A }\end{array}$ & 60 \\
Rated current/A & 13 & 38 \\
\hline
\end{tabular}

\section{Dynamic Simulation Analysis of the Unmanned Platform}

\subsection{The Establishment of dynamic model of unmanned platform}

As is shown in Figure 5, the origin of the platform is $\mathrm{O}_{1}$, $\mathrm{O}_{1}-\mathrm{xy}$ is an absolute Cartesian coordinate system, $\mathrm{C}_{1}$-xy is a relative Cartesian coordinate system, and The origin of the coordinate is the center $\mathrm{C} 1$ of the Mecanum wheel. $\mathrm{V}_{\mathrm{x}}$ is the horizontal speed of the platform while $\mathrm{V}_{\mathrm{y}}$ is the vertical speed. $\omega_{0}$ is the angular speed around the point $\mathrm{O}_{1}$, $\omega_{1} 、 \omega_{2} 、 \omega_{3} 、 \omega_{4}$ are the rotational speeds of the four Mecanum wheels. The point of the absolute motion that relative to the Cartesian coordinate system $\mathrm{O}_{1}$-xy is $\mathrm{C}_{1}$, while Relative to the Cartesian coordinate system $\mathrm{C} 1-\mathrm{xy}$, $\mathrm{C} 1$ does relative motion, thus the relative movement between the two systems is called implicated motion.

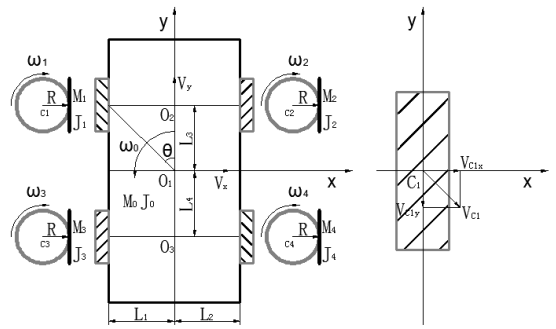

Figure5 Plane view of the unmanned platform

In absolute motion, the speed of point $C_{1}$ in coordinate system $\mathrm{O}_{1}$-xy is

$$
\begin{aligned}
& V_{C 1 x}=V_{x}+\sin \theta L_{1} \omega_{0} \sin \theta=V_{x}+L_{3} \omega_{0} \\
& V_{C 1 y}=V_{y}+\sin \theta L_{1} \omega_{0} \cos \theta=V_{y}+L_{1} \omega_{0}
\end{aligned}
$$


The moving velocity of w1's center $\mathrm{C}_{1}$ in $\mathrm{C}_{1}$-xy system is:

$$
\begin{gathered}
V_{C 1 x}=V_{C 1} \sin \alpha \\
V_{C 1 y}=\mathrm{R} \omega_{1}-V_{C 1} \cos \alpha
\end{gathered}
$$

To sum up:

$$
\begin{gathered}
\mathrm{V}_{\mathrm{x}}+\omega_{0} \mathrm{~L}_{3}=\mathrm{V}_{\mathrm{C} 1} \sin \boldsymbol{\alpha} \\
\mathrm{V}_{\mathrm{y}}+\omega_{0} \mathrm{~L}_{1}=\omega_{1} \mathrm{R}-\mathrm{V}_{\mathrm{C} 1} \cos \boldsymbol{\alpha}
\end{gathered}
$$

Deduce from the (3.5) and (3.6):

$$
\omega_{1}=\frac{1}{\mathrm{R}}\left[\begin{array}{ccc}
\frac{1}{\tan \alpha} & 1 & \frac{L_{3}}{\tan _{\alpha}}
\end{array}\right]\left[\begin{array}{c}
V_{x} \\
V_{y} \\
\omega_{0}
\end{array}\right]
$$

Form these parameter we can get the offset angle $\alpha$ of the roller is $45^{\circ}$. It can be concluded that:

$$
\left[\begin{array}{l}
\omega_{1} \\
\omega_{2} \\
\omega_{3} \\
\omega_{4}
\end{array}\right]=\frac{1}{R}\left[\begin{array}{ccc}
1 & 1 & \left(L_{1}+L_{3}\right) \\
1 & -1 & \left(L_{1}+L_{3}\right) \\
-1 & -1 & \left(L_{1}+L_{3}\right) \\
-1 & 1 & \left(L_{1}+L_{3}\right)
\end{array}\right]\left[\begin{array}{c}
V_{x} \\
V_{y} \\
\omega_{0}
\end{array}\right]
$$

From the above model, it can be seen that changes in the magnitude and direction of $\omega_{1} 、 \omega_{2} 、 \omega_{3} 、 \omega_{4}$ affect the direction and speed of the entire wheeled unmanned platform, which consequently achieve full-scale movement.

\subsection{The Analysis of the motion state of unmanned platform}

According to the above mathematical model, when the platform rotates in situ, that is, the speed in the horizontal and vertical directions is 0 , while the angular velocity is not 0 , the following can be obtained:

$$
\omega_{1}+\omega_{2}+\omega_{3}+\omega_{4}=\frac{L_{1}+L_{3}}{R} \omega_{0}
$$

When the unmanned platform moves back and forth, that is, the horizontal direction and angular velocity is 0 , and the vertical velocity is not 0 , we can get:

$$
\left[\begin{array}{l}
\omega_{1} \\
\omega_{2} \\
\omega_{3} \\
\omega_{4}
\end{array}\right]=\frac{1}{R}\left[\begin{array}{c}
V_{y} \\
-V_{y} \\
-V_{y} \\
V_{y}
\end{array}\right]
$$

Similarly when the unmanned platform moves left and right, that is, the vertical speed and angular speed are 0 , and the horizontal speed is not 0 , then:

$$
\left[\begin{array}{l}
\omega_{1} \\
\omega_{2} \\
\omega_{3} \\
\omega_{4}
\end{array}\right]=\frac{1}{R}\left[\begin{array}{c}
V_{x} \\
V_{x} \\
-V_{x} \\
-V_{x}
\end{array}\right]
$$

When the unmanned platform reaches the left front $45^{\circ}$ or the right front $45^{\circ}$ motion, that means the angular velocity is 0 , and the horizontal and vertical velocities are not 0 , it can be concluded that:

$$
\left[\begin{array}{l}
\omega_{1} \\
\omega_{2} \\
\omega_{3} \\
\omega_{4}
\end{array}\right]=\frac{1}{R}\left[\begin{array}{cc}
V_{x} & V_{y} \\
V_{x} & -V_{y} \\
-V_{x} & -V_{y} \\
-V_{x} & V_{y}
\end{array}\right]
$$

Reviewing the above, the four wheels of the unmanned platform based on the Mecanum wheel can carry out the movement in different directions, as shown in Figure 6:

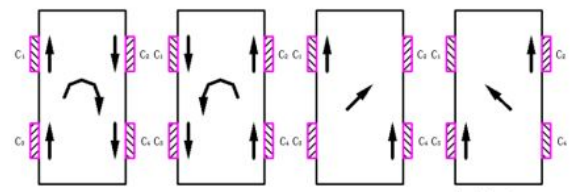

Figure6 platform motion states' diagram

\subsection{Simulation of the Unmanned Platform Based on Adams}

In this paper, we first use UG to simplify the entity model of the unmanned platform, and then import it into the Adams software for simulation. Before the simulation calculation, the parts without motion relations between each other become a rigid body, and remove the rigid body which doesn't participate in the movement and doesn't affect the simulation analysis result. Secondly, ignore the deformation and mechanical errors of the unmanned platform, although it is slightly different from the actual situation, it greatly reduces the computation and it can ensure the accuracy of the calculation.

In the process of simulation, each small roller adds rotation pairs around its axis of rotation, because there are 15 rollers, there are 15 rotation pairs. The Mecanum wheel has 1 rotation pair relative to the unmanned platform, so the total Mecanum wheel has 16 rotation pairs, and the whole platform adds 64 rotation pairs. In addition to adding constraints, we also need to add loads to the model. The loads that need to be added in this simulation are as follows:

1) Add velocity in four Mecanum wheels to simulate motor movements, 4 in total.

2) Add vertical down force to simulate gravity, 1 in total.

3) Apply contact force between various part of the platform, 60 in total.

Simulation of virtual prototype model is shown in Figure 7:

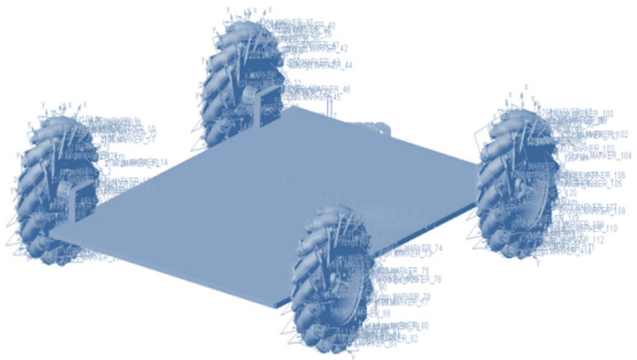

Figure7 Platform Simulation Diagram

\subsection{Analysis of the simulation results of unmanned platform}

This paper analyzes the three motion states of the platform(respectively along $\mathrm{x}, \mathrm{y}$ and $\mathrm{z}$ axis). Simulation duration of all three states is set to $3 \mathrm{~s}$, with 3000 in steps and $\pm 360 \mathrm{rpm}$ of the rotation speed. According to the simulation parameters, the micro wheel rotates three times in total, and the simulation results are calculated under the 
condition of the Mecanum wheel touching the ground and the dynamic friction coefficient is $\mathrm{u}=0.1$ and $\mathrm{u}=0.4$.

(1) Unmanned platform moving around $\mathrm{z}$ axis

As shown in Figure 8 and Figure 9, the Mecanum wheel resistance moment and the platform vibration curve of an unmanned platform moving around the $\mathrm{z}$ axis under different dynamic friction coefficients are as follows:

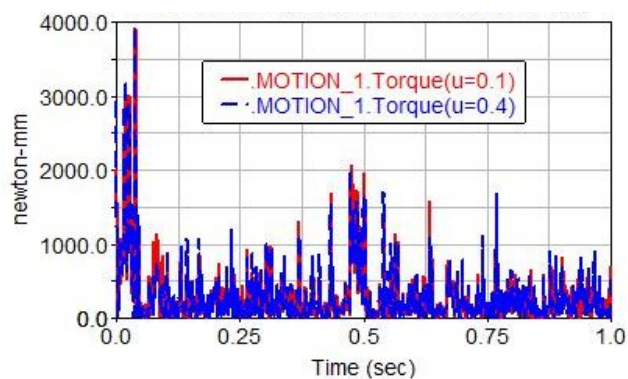

Figure 8 Curve of Resistance Torque Orbiting $\mathrm{z}$ axis

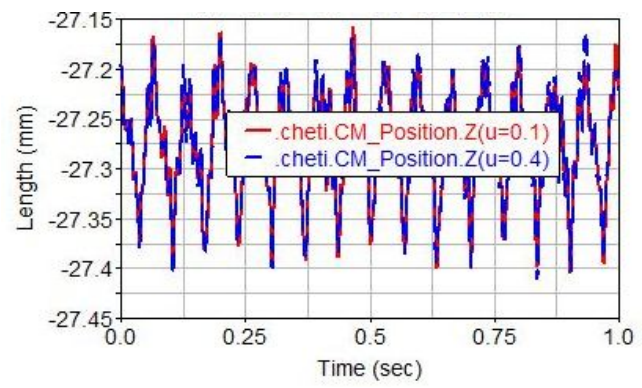

Figure 9 Curve of Vibration Movements Orbiting $\mathrm{z}$ axis

(2) Unmanned platform moving around $\mathrm{x}$ axis

The Mecanum wheel resistance moment and the platform vibration curve of an unmanned platform moving along the $\mathrm{x}$ axis under different dynamic friction coefficients are shown in Figure 10 and 11.:

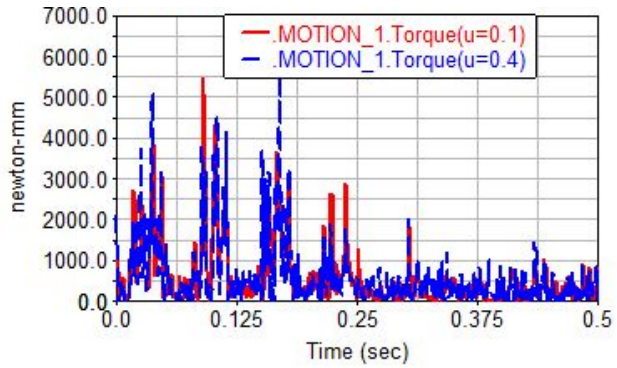

Figure 10 Curve of Resistance Torque

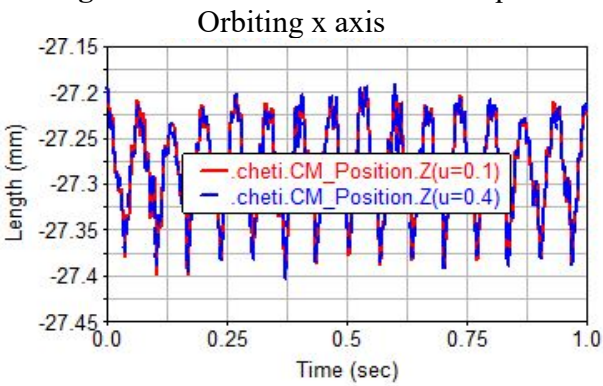

Figure 11 Curve of Vibration Movements Orbiting $\mathrm{z}$ axis

(3) Unmanned platform moving around y axis

Mecanum wheel resistance moments and platform vibration curves moving around $\mathrm{y}$ axis under different dynamic friction coefficients are shown in Figure12 and13:

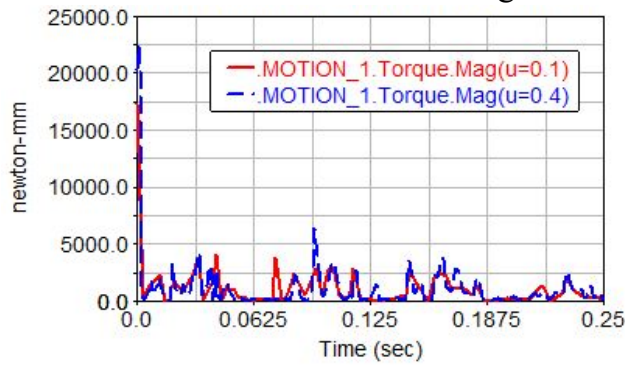

Figure 12 Curve of Resistance Torque Orbiting y axis

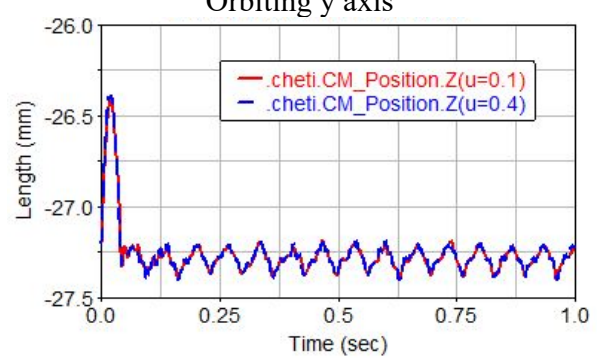

Figure 13 Curve of Vibration Movements Orbiting y axis

From the results of (1), (2), and (3), it can be seen that the same motion state and platform dynamic performance under different dynamic friction coefficients are approximately the same, and the dynamic resistance moment of the platform is always very large at the beginning, and after a period of time, the resistance torque tends to be stable; The amplitudes of the vibrations in all three motion states are not large, indicating that the small unmanned platform based on the Mecanum wheel has good stability in the motion process.

Compared with the traditional differential phasechange unmanned platform, the Mecanum wheel has the advantage of in-situ steering, and the dynamic resistance torque of the wheel is approximately the same as the vibration of the vehicle body and other movement states during the steering process, and there is no excessive peak or instability of the body, and it has good steering performance.

\section{4 summary}

This paper mainly studies the small unmanned platform's motion characteristics of marine Mecanum wheels, and analyzes and compares the motion characteristics in different motion states and different dynamic friction coefficients. Firstly, analyzing the operational requirements of the platform, and the unmanned platform based on the Mecanum wheel was determined as the research object according to the limited working space and narrow working environment; then the three-dimensional simplified model of the unmanned platform was established by using Adams simulation to get the change curve of the wheel resistance moment and platform vibration. And analyze the results to determine the advantages of the unmanned platform based on the Mecanum wheel on the ship's deck. 


\section{References}

1. Modeling and AdaptiveControl of an Omni-MecanumWheeled Robot[J].Lih-ChangLin,Hao-Yin Shih. Intelligent Control and Automation . 2013 (02)

2. Geometry and kinematics of the Mecanum wheel[J]. A. Gfrerrer. Computer Aided Geometric Design . 2008 (9)

3. Visual dead-reckoning for motion control of a Mecanumwheeled mobile robot[J] . J.A Cooney,W.L Xu,G Bright. Mechatronics . 2003 (6) 